\title{
SDCEP launches latest guidance
}

Last month saw the launch of the latest guidance from the Scottish Dental Clinical Effectiveness Programme (SDCEP). The guidance on the Management of Acute dental problems builds on an earlier publication on emergency dental care published in $2007 .{ }^{1}$

The guidance was developed because services for acute dental problems are variable and some problems are rarely encountered and therefore less familiar. In addition some patients seek advice and assistance from other health care providers, and one of the World Health Organisation Oral Health targets for $2020^{2}$ is to increase the number of health care providers who are competent to recognise and manage a range of acute dental problems (infectious diseases, oral mucosal and salivary gland disorders).

The scope of the guidance is to:

- encourage a consistent approach to the management of acute dental problems to reduce avoidable variation in practice

- improve the quality of unscheduled clinical care for patients with acute dental problems

- provide a standard for the initial management of presenting symptoms for patients with acute dental problems

- ensure patients receive appropriate advice about subsequent care and/or referral to appropriate treatment providers, if applicable.

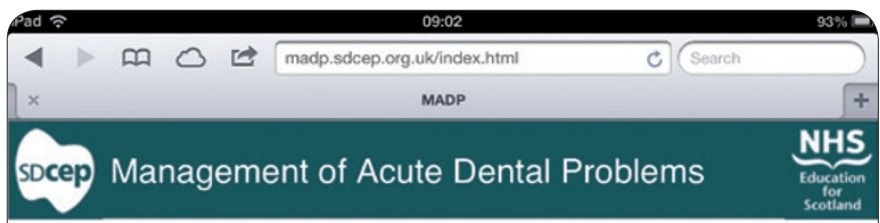

- aprevious answers About Help A-Z of Conditions

\section{i Guidance for healthcare professionals}

Most patients with an acute dental problem have one or more of the six symptoms below. Click on the first reported symptom then follow the subsequent questions to find out about any immediate advice or care you can give and whether the patient is likely to require Emergency, Urgent, Non-Urgent or Self Care.

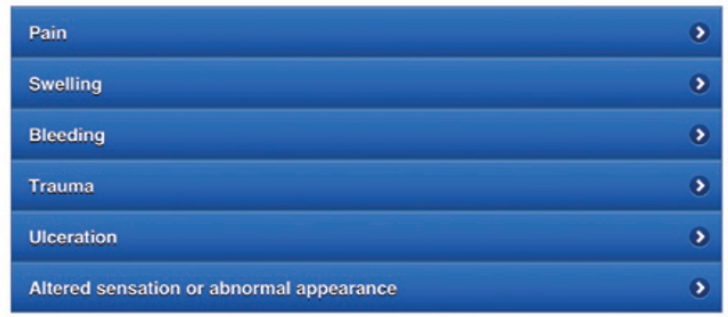

For more advice on the use of this guidance tool, click Help.

To read the Disclaimer, Copyright statement and to find out more about this guidance, click About.

To find guidance on a particular condition, click $\mathbf{A}-\mathbf{Z}$.

This guidance is intended for the management of acute dental problems. If the dental problem is secondary to a more significant problem (e.g. a significant facial injury) or is resulting in severe

Fig 1. Home page of interactive electronic version on tablet

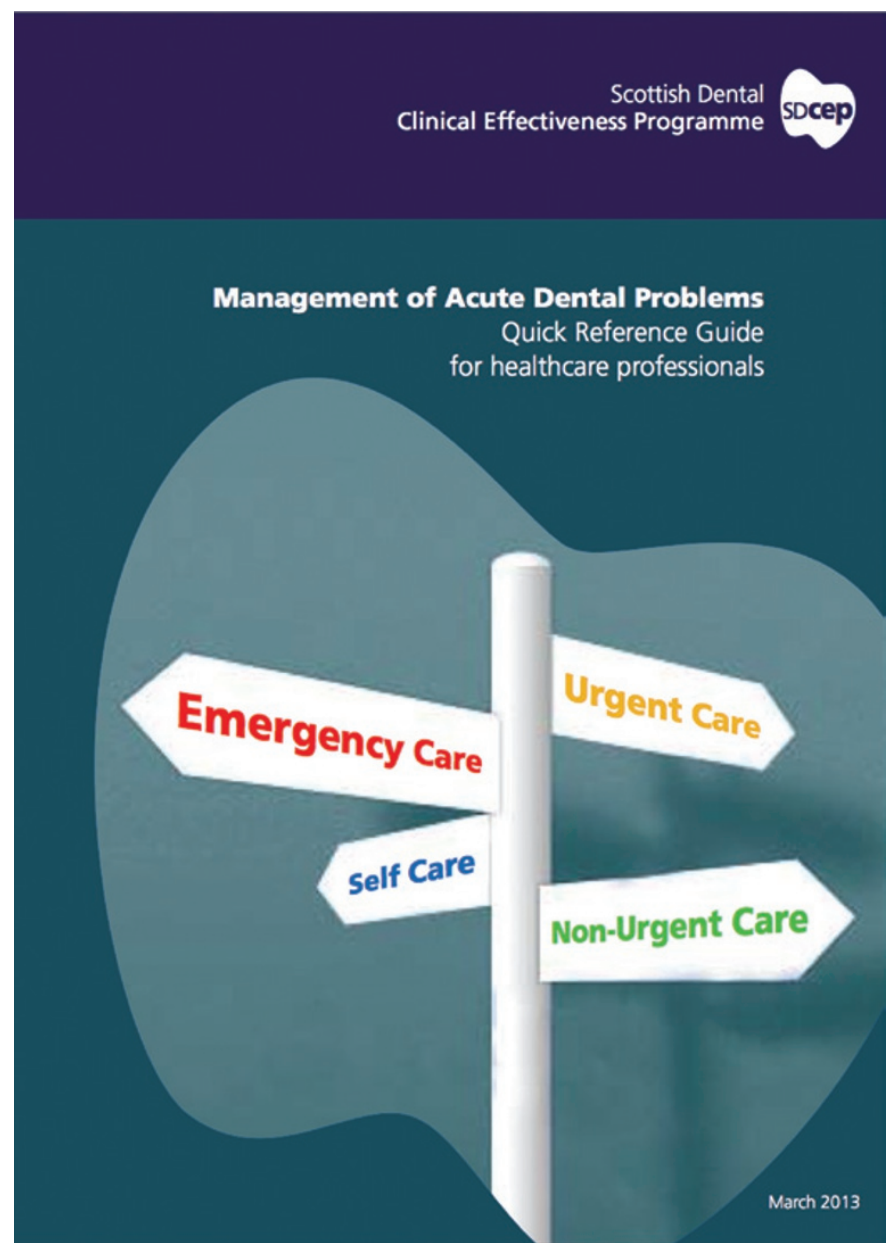

Fig 2. Front cover of the quick reference guide

The guidance has been launched with three main resources:

1. The full guidance document

2. An interactive electronic version of the full guidance

3. A quick reference guide.

The first of these resources, the full guidance document, contains all the usual elements of guidance from SDCEP and is freely available to download from their website. The interactive electronic version (figure 1) is also freely available from www.madp.sdcep.org.uk The electronic version has been developed for use in the current versions of the Firefox, Chrome Safari and Internet Explorer 9 or later. Many NHS organisations are still using Internet Explorer 8 and while the resources does work on browser it is not as visually appealing and there may be some limits to its functionality.

The final resource is a 12-page quick reference guide (Fig. 2). This comprises a brief introduction together with flowcharts related to the main presenting symptoms (pain, swelling, bleeding, trauma, ulceration and altered sensation or appearance). The flowcharts have formed the basis of the electronic version. An example of one of the flow charts is shown in Figure 3. Printed copies of this document are being widely distributed in Scotland to dentists 


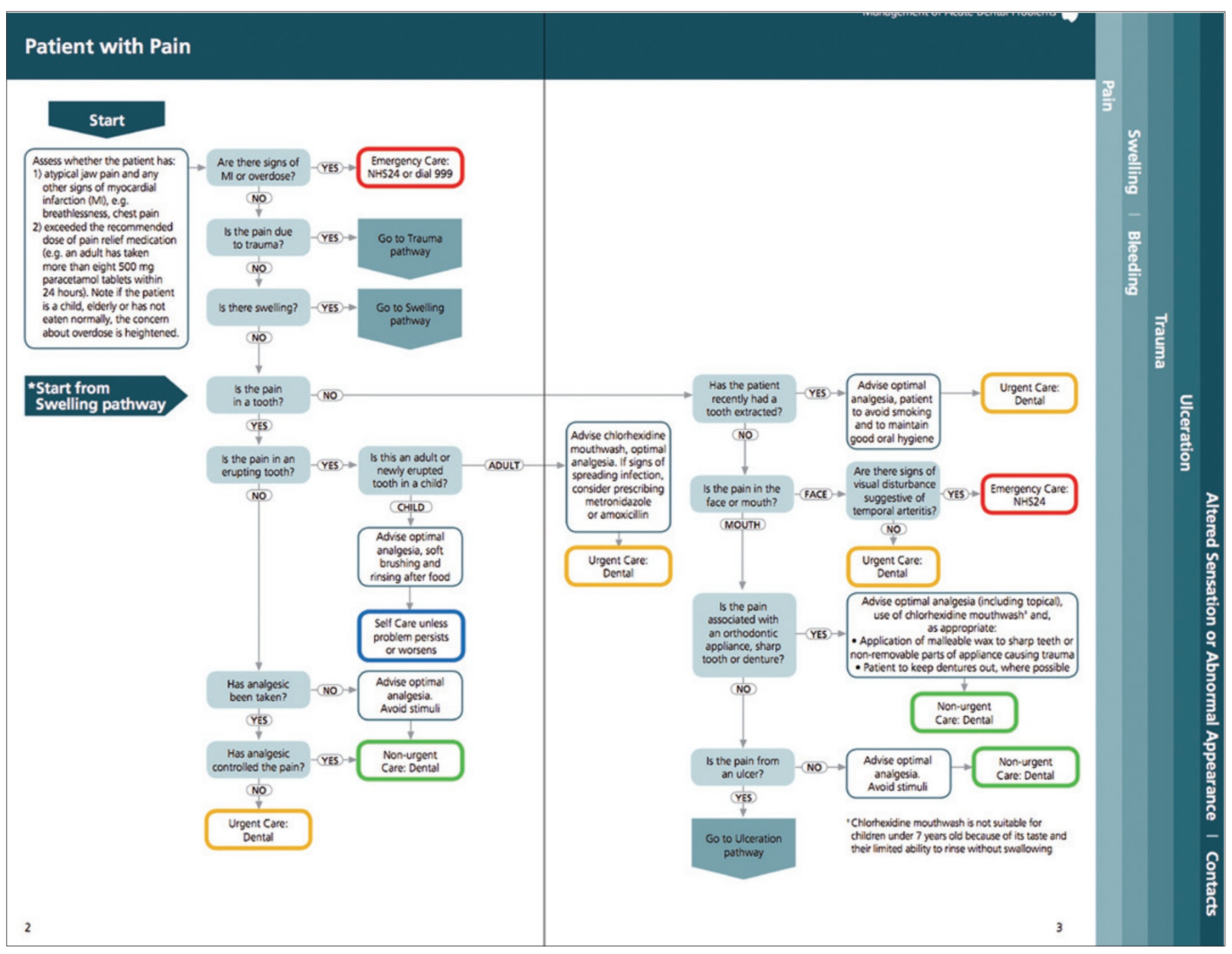

Fig 3. Example flow chart from the quick reference guide

(primary care, salaried, hospital), general medical practices, community pharmacies and emergency departments.

As suggested in the section on who should use this guidance, these resources are being targeted at a wide range of health care professionals rather than specifically at dentists, although they are a key audience. Dentists themselves will probably find the A-Z of conditions section the most useful as this contains very helpful information on initial and subsequent management of dental problems.

However, supporting staff and other healthcare professionals should find the quick reference tool and online guide beneficial in ensuring that patients with acute dental problems receive the most appropriate advice about subsequent care and/or referral to appropriate treatment providers.
The interactive on-line version can be accessed using the tiny URL http://tiny.cc/bylwsw or by scanning the QR code below

1. SDCEP 2007. Emergency dental care: dental clinical guidance. Dundee: Scottish Dental Clinical Effectiveness Programme, 2007 (www.sdcep.org.uk/index. aspx?o=2335).

2. Hobdell M, Petersen PE, Clarkson J, Johnson N. Global goals for oral health 2020. Int Dent / 2003; 53: 285-288.

Evidence-Based Dentistry (2013) 14, 29-30. doi:10.1038/sj.ebd.6400922

Fig 4. QR Code

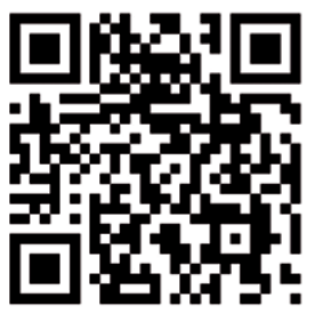

